\title{
TIPOS E QUANTIDADES DE ATIVIDADES FÍSICAS PRATICADAS POR ADOLESCENTES DO SUL DO BRASIL
}

\author{
Marcus Vinicius Veber Lopes \\ Universidade Federal de Santa Catarina, Santa Catarina, Florianópolis, Brasil \\ Kelly Samara da Silva \\ Universidade Federal de Santa Catarina, Santa Catarina, Florianópolis, Brasil \\ Cecília Bertuol \\ Universidade Federal de Santa Catarina, Santa Catarina, Florianópolis, Brasil \\ Jaqueline Aragoni da Silva \\ Universidade Federal de Santa Catarina, Santa Catarina, Florianópolis, Brasil \\ Markus Vinicius Nahas \\ Universidade Federal de Santa Catarina, Santa Catarina, Florianópolis, Brasil
}

\begin{abstract}
Resumo
Objetivou-se estimar as prevalências dos tipos e quantidades de atividade física (AF) no tempo de lazer em adolescentes, por sexo e idade. Estudantes $(15-19$ anos; $n=6.529)$ de escolas estaduais de Santa Catarina responderam a um questionário validado sobre tipos de AF praticadas semanalmente no tempo de lazer. Medidas apresentadas em frequência relativa e intervalos de confiança de $95 \%$ indicaram que, ao comparar os sexos, rapazes apresentaram maior prevalência de prática de futebol, musculação e ciclismo, enquanto moças apresentaram maior prevalência de caminhada, dança e ginástica. Prevalência de caminhada foi maior nos adolescentes de 17 anos, em relação aos de 18 anos. Conclui-se que tipos de AF diferem de acordo com sexo e idade, enquanto a quantidade de AF diferiu entre sexos.
\end{abstract}

Palavras-chave: Atividade Motora; Adolescente; Atividades de Lazer; Estudos Transversais.

\section{TYPES AND QUANTITIES OF PHYSICAL ACTIVITIES PRACTICED BY ADO- LESCENTS FROM SOUTHERN BRAZIL}

\begin{abstract}
The study aimed to estimate the prevalence of types and quantity of physical activity (PA) performed during leisure time by adolescents, according to sex and age. Students (15-19 years; $n=6,529)$ from public schools of Santa Catarina answered a validated questionnaire about types of PA weekly performed during leisure time. Data presented in relative frequency and $95 \%$ confidence interval showed that, when comparing sex, higher prevalence of practice in soccer, weight lifting, and cycling was observed among boys, while walking, dance and gymnastics were more prevalent among girls. The proportion of walking was higher in adolescents aged 17 years old, compared with those aged 18 years old. It is concluded that types of PA differ according to sex and age, whereas the amount of PA differs only by sex.
\end{abstract}

Keywords: Motor Activity; Adolescent; Leisure Activities; Cross-Sectional Studies 


\section{TIPOS Y CANTIDADES DE LAS ACTIVIDADES FÍSICAS PRACTICADAS POR LOS ADOLESCENTES EN EL SUR DE BRASIL}

\section{Resumen}

Se objetivó estimar las prevalencias de los tipos y cantidades de actividad física (AF) en el tiempo libre en adolescentes, por sexo y edad. Los estudiantes (15-19 años, $n=6.529)$ de escuelas estatales de Santa Catarina respondieron a un cuestionario validado sobre tipos de AF practicadas en el tiempo libre. Medidas presentadas en frecuencia relativa e intervalos de confianza del $95 \%$ indicaron que los niños presentaron mayor prevalencia de práctica de fútbol, musculación y ciclismo, mientras que las niñas practican más la caminata, la danza y la gimnasia. La prevalencia de caminata fue mayor en los adolescentes de 17 años, en relación a los de 18 años. Se concluye que los tipos de AF difieren según el sexo y la edad, mientras que la cantidad de AF se diferenció entre los sexos.

Palabras clave: Actividad Motora; Adolescente; Actividades Recreativas; Estudios Transversales

\section{Introdução}

A participação em atividades físicas (AF) oferece diversos benefícios à saúde do adolescente, sejam de caráter físico (JANSSEN; LEBLANC, 2010; LANDRY; DRISCOLL, 2012); psicossocial (LANDRY; DRISCOLL, 2012; LUZ; KUHNEN, 2013); ou comportamental, como a melhora da qualidade do sono, hábitos alimentares adequados e redução do comportamento sedentário (BARBOSA FILHO, 2012). Esta participação pode se manifestar em diferentes contextos do cotidiano do adolescente, caracterizados como os domínios doméstico, ocupacional/escolar, de deslocamento e de lazer (BAUMAN et al., 2012; NAHAS, 2017). Entende-se o contexto do lazer por um conjunto de ocupações que possuem como finalidade o descanso, o divertimento ou, ainda, o desenvolvimento pessoal e social, sendo desenroladas no momento em que o indivíduo está livre de suas obrigações familiares, profissionais e sociais (DUMAZEDIER, 1979; CAMARGO, 2016). Não obstante, em adolescentes, o domínio do lazer representa um grande componente do tempo diário que poderia ser destinado ao engajamento em AF (ESCULCAS; MOTA, 2005).

Estudo de revisão sistemática que tratava sobre as prevalências de AF no Brasil verificou que, dentre os 47 estudos incluídos, a maior parte investigava o comportamento ativo no domínio do lazer, o que sugere seu importante papel na contribuição para a prática de AF em níveis adequados para a aquisição de benefícios à saúde (DUMITH, 2009). Contudo, investigações indicam que, mesmo avaliando os diferentes domínios da AF, a prevalência de inatividade física tem aumentado nos últimos anos, sobretudo na população jovem (DUMITH et al., 2011). A prática de AF na adolescência deve ser estimulada, uma vez que jovens fisicamente ativos, ou que se engajam em atividades esportivas (KOORTS et al., 2011), tendem a manter o comportamento ativo na vida adulta (ALVES et al., 2005; AZEVEDO JUNIOR; ARAÚJO; PEREIRA, 2006; CORDER et al., 2017). Além de promover a prática, é essencial que sejam considerados os tipos de atividades e as diferenças entre os sexos e as idades (WOLL et al., 2011) quanto à adoção desse comportamento. A caracterização dessas diferenças, em dada população, permite a compreensão de lacunas que permanecem vigentes na literatura, como a identificação do perfil de prática e dos interesses dos adolescentes.

Quando investigado os tipos de AF mais populares, observa-se que estes diferem consideravelmente entre as regiões estudadas - como o ciclismo na Noruega (KJØNNIKSEN; TORSHEIM; WOLD, 2008), a caminhada no Canadá (BELANGER et al., 2009), e o voleibol nos Estados Unidos (GRIESER et al., 2006; BUTTE et al., 2007). No Brasil, apesar de haver uma diversidade de tipos de AF praticadas, percebe-se que o futebol é o tipo mais frequente 
entre os rapazes (AZEVEDO JUNIOR; ARAÚJO; PEREIRA, 2006; DUMITH et al., 2010) e o voleibol, a caminhada e a dança, entre as moças (AZEVEDO JUNIOR; ARAÚJO; PEREIRA, 2006; DUMITH et al., 2010; MATIAS et al., 2012). Em relação à faixa etária, parece existir uma tendência de redução na participação em atividades coletivas com o decorrer da idade, enquanto a prática de atividades de caráter individual é mantida (BELANGER et al., 2009). Outro aspecto importante é que os padrões de atividades parecem ser influenciados por tradições culturais e condutas impostas pela sociedade, as quais divergem entre sexos e idades (ROLEY et al., 2008), sobretudo em países com grande diversidade cultural, como é o caso do Brasil (OLIVEN, 2011).

Além dos tipos de AF praticados, a investigação da quantidade de atividades nas quais os jovens se engajam parece assumir papel importante no aumento da adesão e manutenção da prática. Evidências indicam que o aumento dos níveis de AF - reflexo da participação em atividades - é dado primariamente em função da quantidade de atividades praticadas e, então, da frequência e do volume (AARON et al., 2002; DUMITH et al., 2012a) das mesmas. Portanto, a prática de diferentes tipos de AF deve ser encorajada entre os adolescentes.

Sabe-se que, ao longo das últimas décadas, adolescentes têm reduzido o tempo em trabalho remunerado e disponibilizado mais tempo para atividades de lazer (ZICK, 2010), o que pode favorecer o engajamento em práticas esportivas. No entanto, ainda são poucos os estudos que investigaram quantos e quais são os tipos de AF praticados pelos adolescentes neste domínio, o que poderia auxiliar no desenvolvimento de programas de promoção de hábitos fisicamente ativos (DUMITH et al., 2012a; MATIAS et al., 2012). Dado o exposto, este estudo tem por objetivo estimar as prevalências dos tipos e das quantidades de AF realizadas no tempo de lazer por adolescentes de escolas públicas estaduais de Santa Catarina; bem como, identificar as diferenças entre os sexos e idades dentre as atividades mais praticadas.

\section{Métodos}

Este estudo trata-se de um segmento da pesquisa "Estilo de vida e comportamentos de risco de jovens catarinenses - CompAC (Comportamentos dos Adolescentes Catarinenses)", com característica epidemiológica de base escolar, abrangência estadual e delineamento transversal, realizada em 2011. O estudo investiga o estilo de vida de estudantes do ensino médio com idades entre 15 e 19 anos $(16,3 \pm 1,0)$ de escolas públicas estaduais de Santa Catarina.

O cálculo amostral considerou prevalência desconhecida do fenômeno investigado de $50 \%$, com intervalo de confiança de $95 \%$ e erro máximo de dois pontos percentuais. Os parâmetros aplicados resultaram em uma amostra mínima de 2.373 adolescentes, duplicada em virtude de a amostra ser por conglomerados $(n=4.746)$. A amostra foi ampliada em $25 \%$ considerando possíveis casos de perdas e recusas, o que resultou em um tamanho amostral final estimado em 5.932 adolescentes. Ao final do processo, 6.529 estudantes participaram do estudo. Para distribuição da amostra considerou-se como unidade primária de amostragem o porte das escolas (pequeno: $<200$ estudantes, médio: 200-499, grande $\geq 500$ ), e como unidade secundária, as turmas, estratificadas por turno (diurno e noturno) e ano escolar $\left(1^{\circ}\right.$ ao $3^{\circ}$ ano do ensino médio). Outras informações poderão ser consultadas em trabalho já publicado (SILVA, K. et al., 2013).

Como instrumento de pesquisa, utilizou-se o questionário COMPAC, previamente adaptado e validado para a população alvo, com aceitável reprodutibilidade $(0,51-0,96)$ e validade de face e conteúdo (DE BEM et al., 2000). A diversidade e os tipos de AF praticados no tempo de lazer, variáveis desfecho do presente estudo, foram obtidos por meio do preenchimento de um quadro contendo 19 AF comuns aos adolescentes de Santa Catarina. Na mesma questão havia duas outras alternativas: a) a possibilidade de incluir alguma atividade 
não descrita dentre as opções; e b) opção referente à não prática de AF no tempo de lazer. $\mathrm{O}$ preenchimento do quadro era guiado pelo aplicador, o qual instruía os adolescentes a marcarem todas as atividades praticadas exclusivamente no domínio de lazer em uma semana habitual, desconsiderando aquelas realizadas no período escolar obrigatório, no trabalho e no deslocamento. As informações quanto ao sexo e à idade dos adolescentes foram coletadas por meio do mesmo instrumento.

As análises estatísticas foram conduzidas no software estatístico Stata Standard Edition 13. Foram utilizadas análises descritivas com frequências absolutas e relativas, e intervalos de confiança de $95 \%$ para a comparação entre as variáveis.

O projeto foi aprovado pelo Comitê de Ética em Pesquisas com Seres Humanos da Universidade Federal de Santa Catarina sob o parecer de no: 1029/2010. Como critério para participação do estudo, foi requerida a autorização dos adolescentes (a partir de 18 anos) e de seus responsáveis (<18 anos), mediante aplicação do Termo de Consentimento Livre e Esclarecido.

\section{Resultados}

Do total de 6.529 participantes, 6.489 responderam às questões investigadas. Destes, a maior parte era do sexo feminino $(55,5 \%)$ e composta por adolescentes mais jovens, com idades de $15(26,6 \%)$ e 16 anos $(34,1 \%)$. A proporção de adolescentes mais velhos se distribuiu entre as idades de $17(29,8 \%), 18(7,6 \%)$ e 19 anos $(1,9 \%)$.

As atividades com maior prevalência de participação entre os rapazes foram o futebol (58,7\%), a caminhada (16,6\%), a musculação (16,3\%) e o ciclismo (14,3\%); já entre as moças, a caminhada $(32,7 \%)$, o futebol $(12,1 \%)$, a dança $(12 \%)$ e atividades com ênfase em alongamentos, como o yoga e tai chi chuan $(10,2 \%)$. Quando confrontados os sexos, a análise indicou diferenças entre rapazes e moças em relação às prevalências dos tipos de AF praticadas (Figura 1). Observou-se proporção superior dos rapazes, comparados às moças, na participação das práticas de futebol (58,7\%; IC95\%: 55,7; 61,6 versus 12,1\%; IC95\%: 9,8; 14,9), musculação $(16,3 \%$; IC95\%: 14,5; 18,2 versus 7,6\%: IC95\%: 5,8; 9,9) e ciclismo $(14,3 \%$; IC95\%: 13,4; 16,4 versus 5,4\%, IC95\%: 4,3; 6,8). As atividades com maior prevalência de participação das moças, em comparação aos rapazes, foram a caminhada $(32,7 \%$, IC95\%: $29,3$; 36,4 versus $16,6 \%$; IC95\%: 14,$2 ; 19,4)$, a dança $(12,0 \%$; IC95\%: 10,$4 ; 13,9$ versus 3,3\%; IC95\%: 2,7; 4,1) e a ginástica (5,0\%; IC95\%: 3,9; 6,5 versus 0,8\%; IC95\%: 0,$4 ; 1,5)$.

Ao confrontar as idades quanto à participação em diferentes tipos de AF (Figura 2) identificou-se o futebol como a atividade com maior prevalência de participação em todas as idades investigadas, com proporção aproximada de 35\%. As prevalências de participação na caminhada foram de aproximadamente $27 \%$ entre as idades de 15,16 e 17 anos, e observouse um decréscimo significativo na proporção de praticantes ao comparar o grupo de 17 anos (26,2\%. IC95: 22,5; 30,2) com o de 18 anos de idade (14,7\%, IC95: 10,7; 19,9). Percebeu-se uma redução gradual na participação em atividades com ênfase em alongamento com o decorrer da idade, embora sem significância estatística. A prática de musculação apresentou prevalências de participação entre $10 \%$ e $15 \%$, enquanto a proporção de participação nas atividades ciclismo, voleibol e dança se manteve próxima de 10\%, também sem diferir significativamente entre as idades. As práticas de tênis de mesa, ginástica de academia e pesca recreativa apresentaram prevalências de aproximadamente $5 \%$ em todas idades, de forma que as demais basquete, handebol, surfe, tênis de quadra, judô, natação, boliche e capoeira - foram pouco praticadas, com proporções inferiores a $3 \%$. Informações complementares referentes às prevalências de participação por idades estão disponíveis como material suplementar 
Figura 1 - Diferenças entre rapazes e moças quanto aos tipos de atividades físicas praticadas no tempo de lazer. Santa Catarina, $2011(n=6.489)$.

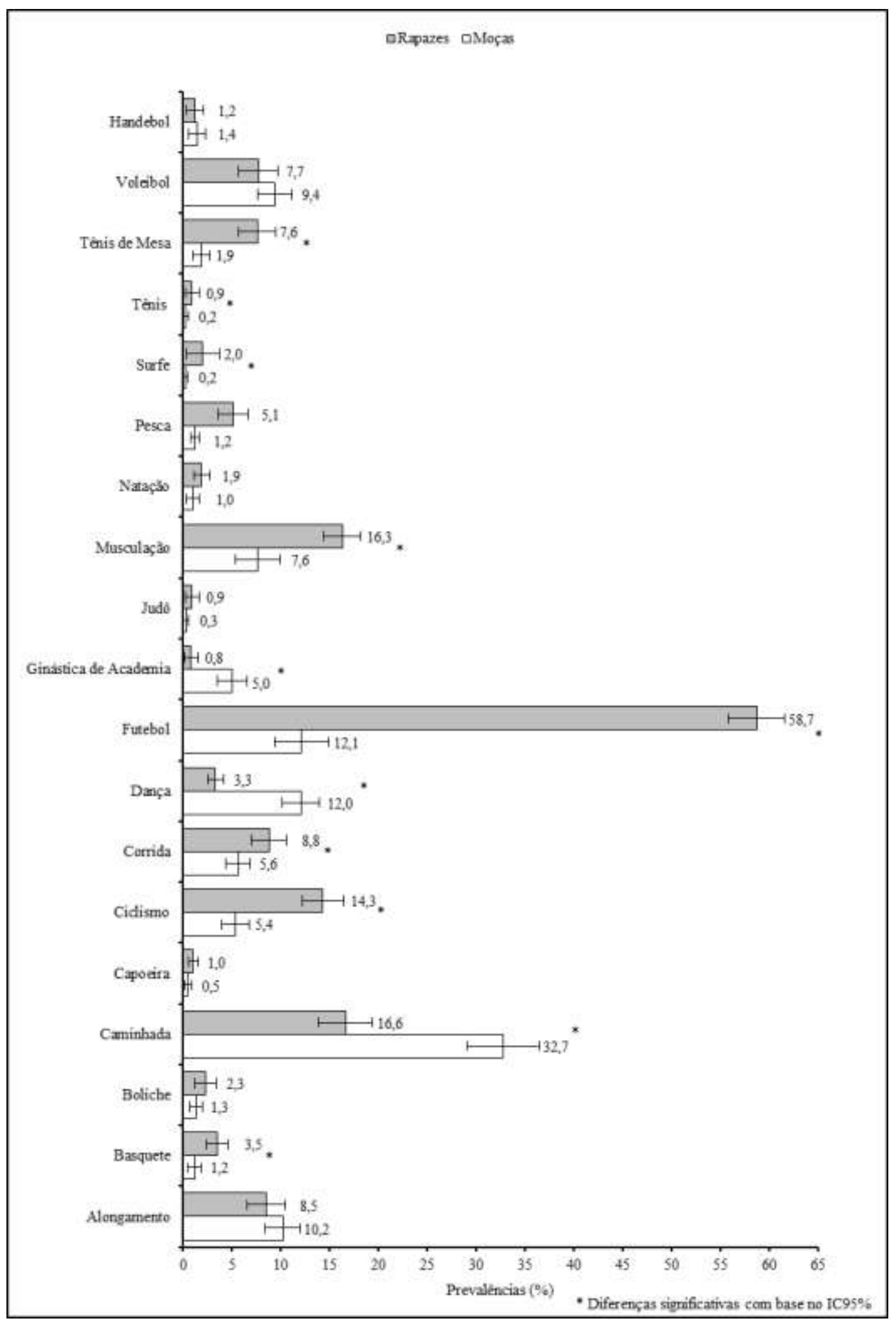

Fonte: Elaborado pelo autor, 2018. 
Figura 2 - Diferenças entre as idades quanto aos tipos de atividades físicas praticadas por adolescentes no tempo de lazer. Santa Catarina, $2011(n=6.489)$.

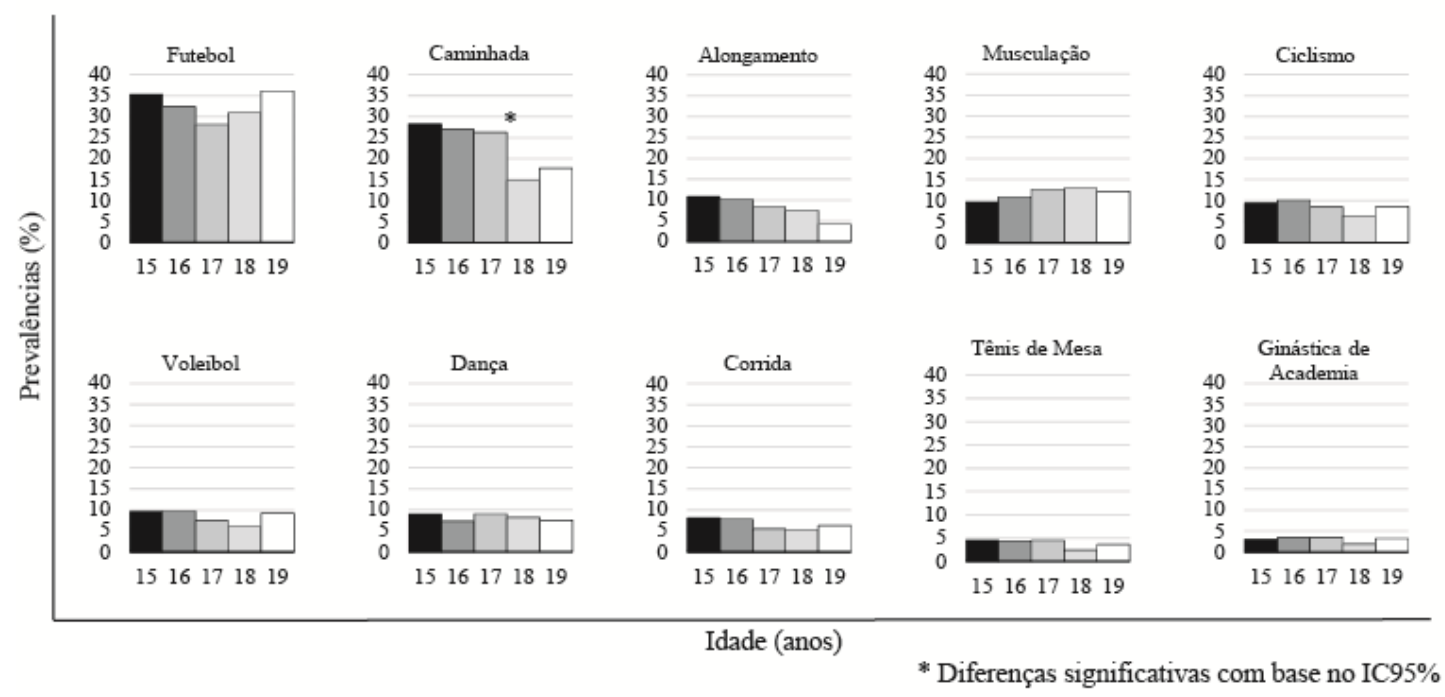

Fonte: Elaborado pelo autor, 2018.

Quando analisada a quantidade de AF praticadas no tempo de lazer, observou-se que quanto maior o número de $\mathrm{AF}$ praticadas, menores as prevalências de praticantes, em rapazes e moças (Figura 3). A prática de um tipo de AF (39,2\%, IC95\%: 36,6; 42,2 versus 29,3, IC95\%: 27,9; 30,8), dois (23,6\%, IC95\%: 21,6; 25,7 versus 17,1\%, IC95\%: 15,1; 19,4), três $(13,0 \%$, IC95\%: 11,$3 ; 14,8$ versus $7,6 \%$, IC95\%: 6,$5 ; 8,9)$ e quatro ou mais tipos de atividades (9,5\%, IC95\%: 7,9; 11,4 versus 5,5\%, IC95\%: 4,3; 7,1) foram superiores entre os rapazes, em relação às moças. As moças apresentaram maior prevalência apenas de não prática de $\mathrm{AF}$ (40,3\%, IC95\%: 37,0; 43,8) em comparação aos rapazes $(14,7 \%$, IC95\%: 13,0; 16,6).

Figura 3 - Diferenças entre rapazes e moças quanto à quantidade de atividades físicas praticadas no tempo de lazer. Santa Catarina, $2011(\mathrm{n}=6.489)$.

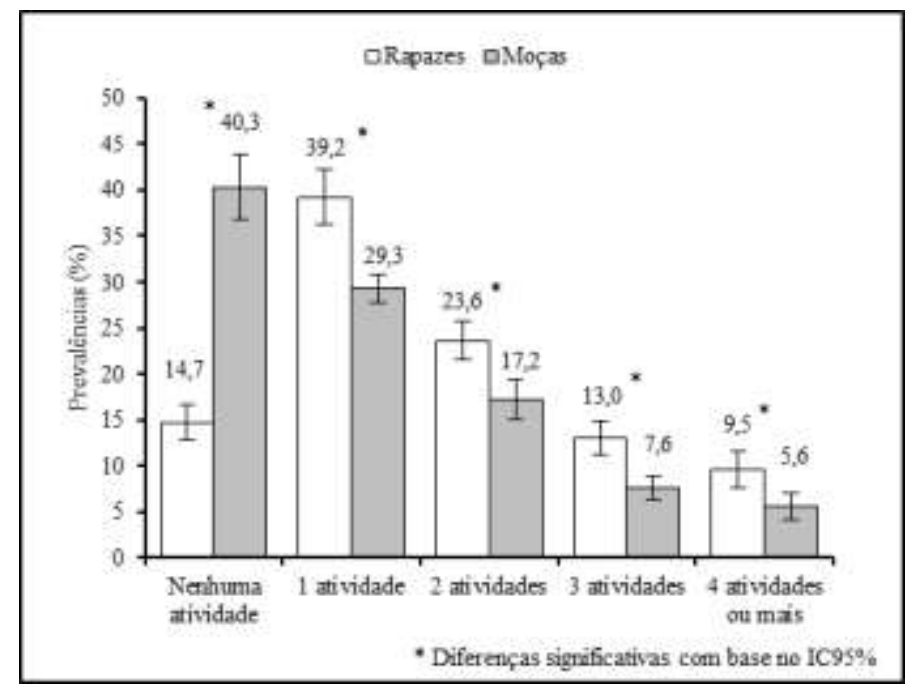

Fonte: Elaborado pelo autor, 2018. 
Em relação às idades, não foram identificadas diferenças estatisticamente significativas quanto à quantidade de atividades praticadas (Figura 4). Ainda, dos adolescentes que praticavam AF no tempo de lazer, aproximadamente $50 \%$ variavam os tipos de AF praticados, participando de dois ou mais tipos distintos de atividades; não havendo diferenças estatisticamente significativas entre as idades.

Figura 4 - Diferenças entre as idades quanto à quantidade de atividades físicas praticadas por adolescentes no tempo de lazer. Santa Catarina, $2011(n=6.589)$.

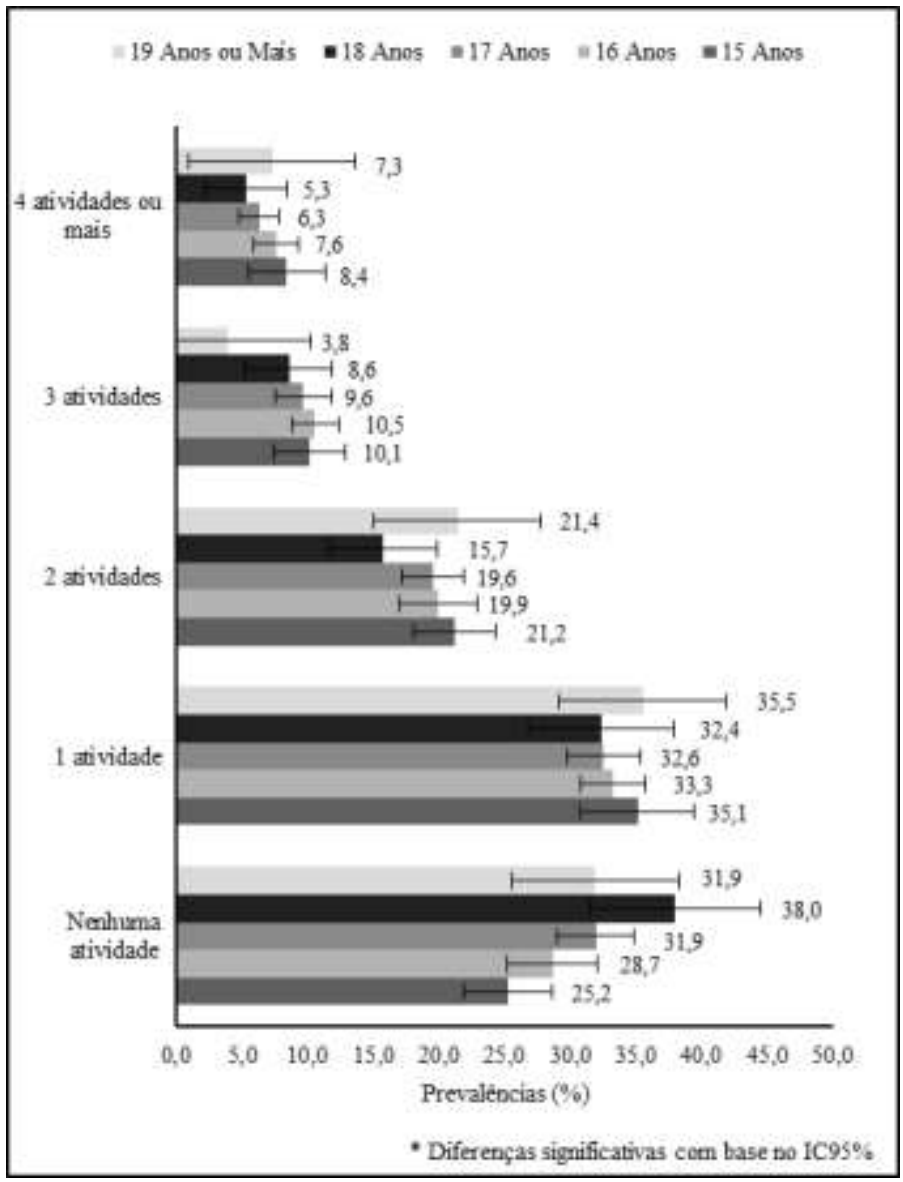

Fonte: Elaborado pelo autor, 2018.

\section{Discussão}

O presente estudo investigou as prevalências de participação em diferentes tipos e quantidades de AF em adolescentes catarinenses. Percebeu-se maior concentração das proporções de prática no futebol e na caminhada, em ambos os sexos e em todas as idades. Ainda, aproximadamente $50 \%$ dos jovens que praticam AF no tempo de lazer participam de apenas um tipo de atividade.

Considera-se a prática de AF como um comportamento complexo, multidimensional e determinado por fatores biológicos, psicossociais, socioculturais e ambientais (STERDT; LIERSCH; WALTER, 2014), que vem sendo investigado utilizando-se de diversos modelos teóricos (RHODES; NIGG, 2011). É evidenciado que o suporte social tem papel fundamental na adoção e manutenção da AF na adolescência (STERDT; LIERSCH; WALTER, 2014). Observa-se que a família parece ser o principal agente socializador na transmissão de valores, comportamentos e normas na infância (SEABRA et al., 2008). Estes comportamentos tendem a ser conduzidos até a adolescência, mesmo este período caracterizando-se por um maior sen- 
timento de autonomia e independência (SEABRA et al., 2008). A escola representa também um contexto relevante na formação do apreço pela prática de AF, sobretudo em função da Educação Física Escolar, que tem um importante papel em incentivar e oportunizar vivências em diferentes práticas (MORTON et al., 2016). Não obstante, é pertinente ressaltar que a adesão à prática é também condicionada a fatores socioeconômicos e ambientais (STERDT; LIERSCH; WALTER, 2014). O engajamento em determinados tipos de AF é muitas vezes limitado em decorrência da dificuldade de acesso aos locais de prática e do maior investimento financeiro para seu o início e manutenção.

A influência de tais aspectos socioculturais na prática de AF pode vir a diferir entre determinados subgrupos da população. Neste estudo, observou-se que as opções de AF realizadas no tempo de lazer diferem entre rapazes e moças. É importante considerar que a adoção de comportamentos distintos pode estar atrelada a aspectos socioculturais que permeiam o papel do gênero no auto-conceito em adolescentes. Segundo o modelo teórico proposto por Athenstaedt (2003) e Athenstaedt e colaboradores (2009), o indivíduo considera determinados comportamentos como tipicamente masculinos ou femininos, bem como características expressivas e instrumentais socialmente desejáveis ou indesejáveis na criação de sua identidade. No que se refere às opções de lazer, as moças valorizam atividades de cuidado pessoal, refletindo a importância dada à autoimagem feminina na sociedade, assim como atividades de socialização (PFEIFER; MARTINS; SANTOS, 2010). Os rapazes, por sua vez, têm maior preferência por atividades esportivas e voltadas à tecnologia (ATHENSTAEDT; MIKULA; BREDT, 2009). Nesse sentido, a exposição a determinados comportamentos e atitudes expressos na sociedade pode influenciar a adoção de tais comportamentos por parte dos jovens, incluindo o engajamento em atividades esportivas. Identificar as preferências e práticas mais prevalentes entre os adolescentes permite uma melhor compreensão de como estereótipos de gênero se manifestam na $\mathrm{AF}$, e ainda possibilita o direcionamento de estratégias para a promoção de hábitos fisicamente ativos nessa população.

O futebol se destacou como atividade com maior participação entre os rapazes, seguido da musculação e do ciclismo, quando comparados às moças. Mesmo diante das diferenças culturais presentes no Brasil (OLIVEN, 2011), estudos nacionais identificaram o futebol como o tipo de AF mais praticado em determinadas regiões (AZEVEDO JUNIOR; ARAÚJO; PEREIRA, 2006; DUMITH et al., 2010; FREITAS et al., 2010; MATIAS et al., 2012). Devido à sua popularidade e por fazer parte da cultura brasileira (SALLES-COSTA et al., 2003; FARIA, 2014), os espaços necessários para a prática do futebol são bastante frequentes (SILVA, I. et al., 2015) e fáceis de serem adaptados, além de ser uma modalidade incentivada desde a infância entre os meninos (SALLES-COSTA et al., 2003). Já a participação na musculação e no ciclismo pode ser explicada por determinados fatores socioculturais (BARBOSA FILHO, 2016). Os rapazes são criados com maior autonomia e possuem maior liberdade para sair de casa, favorecendo a prática de atividades como o ciclismo (SEABRA et al., 2008; BARBOSA FILHO, 2016).

A caminhada foi identificada como a atividade mais praticada entre as moças, seguida pela dança e a ginástica, em relação aos rapazes. Outros estudos evidenciaram a popularidade da caminhada, tanto em território nacional (FREITAS et al., 2010; MATIAS et al., 2012; SOUSA, 2012), quanto internacional (GRIESER et al., 2006; BUTTE et al., 2007; KJØNNIKSEN; TORSHEIM; WOLD, 2008; BELANGER et al., 2009; SIGMUNDOVA et al., 2011). Estudo conduzido com adolescentes (PIRES et al., 2004) constatou que, comparando-se os sexos, as moças realizam mais atividades de baixa intensidade (como a caminhada), enquanto os rapazes, atividades intensas. De acordo com Malta e colaboradores (2010), culturalmente, as moças são expostas a atributos e papéis sociais em sua infância que poderiam influenciar o tipo de prática adotado. Enquanto são estimuladas a se envolverem em atividades mais leves - como brincar de boneca e auxiliar nos afazeres domésticos -, os rapazes 
são motivados a participarem de AF vigorosas, como aquelas de caráter esportivo (MALTA et al., 2010; BARBOSA FILHO, 2016). Em relação à prevalência superior da participação em ginástica entre as moças, acredita-se ser um reflexo do aumento da quantidade de academias de ginástica como atividade comercial, que se dá em conjunto da ampliação dos conhecimentos a respeito dos benefícios da prática de AF para a saúde (AZEVEDO JUNIOR; ARAÚJO; PEREIRA, 2006).

A musculação esteve entre as atividades mais praticadas pelos adolescentes, embora com maior participação dos rapazes. A participação em ginástica de academia, por sua vez, foi predominante entre as moças. Sabe-se que ambas as atividades são popularmente promovidas como meios de se adquirir benefícios estéticos e para a saúde. A preocupação com o corpo é recorrente tanto em rapazes quanto em moças, de forma que os padrões estéticos a serem seguidos têm forte influência da mídia (RODGERS; MCLEAN; PAXTON, 2015; LIRA et al., 2017), sobretudo com a ampliação dos veículos midiáticos devido à inclusão digital e acesso a novas tecnologias. Contudo, evidenciam-se mudanças nos ideais estéticos, que diferem entre sexos e regiões (MELLOR et al., 2013, 2014). Em estudo realizado com adolescentes do Sul do Brasil, observou-se que rapazes são mais insatisfeitos com a magreza, enquanto as moças com o excesso de peso (DUMITH et al., 2012b). Outros estudos identificaram que a insatisfação corporal de rapazes relaciona-se principalmente com o desejo de ganhar peso e musculatura (GONÇALVES; MARTÍNEZ, 2014; MELLOR et al., 2014), enquanto nas moças com o desejo de perder peso (GONÇALVES; MARTÍNEZ, 2014) ou ter uma silhueta menor (LIRA et al., 2017). Nesse sentido, é possível que os padrões de corpos idealizados pelos adolescentes tenham um papel fundamental no engajamento em atividades promovidas com fins estéticos. A musculação tem sido bastante promovida como atividade voltada ao aumento da massa magra; já a ginástica de academia é comumente promovida como de caráter aeróbio, o que pode remeter a atividades voltadas à redução de peso.

A prevalência de não participação em AF foi maior entre moças em comparação aos rapazes, achados estes que corroboram com a literatura (AZEVEDO JUNIOR; ARAÚJO; PEREIRA, 2006; DUMITH et al., 2010; DIAS et al., 2014). Os rapazes apresentam maior autodeterminação (MARKLAND; INGLEDEW, 2007) e autoeficácia (DISHMAN et al., 2005) para a prática de AF, dessa forma, dispõem de maior motivação intrínseca e percebem menos barreiras para a realização desse comportamento. As moças, por sua vez, apresentam maior sentimento de obrigação com essas atividades (MARKLAND; INGLEDEW, 2007) e são menos tolerantes aos desconfortos resultantes da prática, como o risco de lesão e presença de sudorese (GRIESER et al., 2006). Contatou-se também que, em ambos os sexos, houve uma redução nas prevalências de participação à medida que aumentou a quantidade de AF. Entretanto, a proporção de rapazes que participaram de até três tipos de AF foi superior à das moças. Estes achados corroboram com a literatura (DUMITH et al., 2012a) e demonstram a pouca aceitabilidade das moças em engajar em um maior número de práticas.

Com exceção da caminhada, as prevalências de atividades praticadas não apresentaram diferenças de acordo com a idade, o que vai de encontro com evidências prévias (BRINGOLF-ISLER et al., 2009; WOLL et al., 2011; MCMULLAN et al., 2012). Adolescentes mais velhos praticam menos AF em comparação aos mais novos (SANTOS et al., 2005; BUTTE et al., 2007; ORTEGA et al., 2013), principalmente devido às obrigações emergentes no que se refere à maior demanda de estudo e entrada no mercado de trabalho (MATIAS et al., 2012). É importante considerar que, neste estudo, a amostra investigada compreendia uma faixa etária reduzida, entre 15 e 19 anos, enquanto outros estudos compararam jovens mais velhos com aqueles no início da adolescência (ESCULCAS; MOTA, 2005; SANTOS et al., 2005; BUTTE et al., 2007).

Observou-se que a prevalência de participação não diferiu entre as idades para as categorias de prática de uma a quatro ou mais atividades. Este resultado vai de encontro à literatu- 
ra, dado que estudos têm encontrado uma redução no número de atividades praticadas, sobretudo entre as moças (AARON et al., 2002; DUMITH et al., 2012a). Evidências apontam que há um aumento dos níveis de AF à medida que aumentava a quantidade de atividades praticadas no tempo de lazer (DUMITH et al., 2010, 2012a). De acordo com Aaron e colaboradores (2002), o declínio dos níveis de AF durante a adolescência se dá, primeiramente, devido à redução do número de atividades praticadas. Estes achados reafirmam a necessidade de possibilitar aos jovens o engajamento em diversas atividades de lazer.

Diante do exposto, o presente estudo apresentou contribuições relevantes a respeito do perfil de prática de AF dos adolescentes catarinenses, até então pouco explorados na literatura. Dentre os pontos fortes, destacam-se a seleção e o tamanho da amostra, bem como sua representatividade. Foi abordado um tema escasso na literatura nacional, ainda que de considerável importância para a promoção da $\mathrm{AF}$, de forma a ser o primeiro estudo a fornecer $\mathrm{o}$ perfil de prática de estudantes de escolas públicas de um estado brasileiro. Embora forneça medidas subjetivas, o questionário utilizado foi previamente validado para a população investigada.

No entanto, ainda que este estudo tenha apresentado informações expressivas para a área de $\mathrm{AF}$, seus resultados devem ser interpretados com cautela, visto que existem algumas limitações. A amostra do estudo inclui apenas estudantes de escolas estaduais de Santa Catarina e, portanto, seus dados não são representativos de estudantes de escolas particulares ou de adolescentes que não frequentavam a escola na época.

\section{Conclusão}

No que se refere aos tipos de AF, observou-se que os rapazes praticam mais o futebol, o ciclismo e a musculação; enquanto as moças, a caminhada, a dança e a ginástica. Para a idade, apenas a prática da caminhada apresentou redução na proporção de participantes dos 17 para os 18 anos. Observou-se que quanto maior o número de AF praticadas, menores as prevalências de praticantes. A proporção de moças que não participam de nenhuma AF foi superior à dos rapazes, enquanto estes apresentaram prevalências de prática superiores para uma ou mais quantidades de AF no tempo de lazer. Não foram encontradas diferenças significativas quando comparados estudantes de diferentes idades quanto à participação em uma ou mais atividades no tempo de lazer.

Os resultados deste estudo podem contribuir para o fomento de políticas públicas de promoção de saúde, bem como para a elaboração de pesquisas e programas voltados à adesão e aderência à prática de $\mathrm{AF}$ por adolescentes. É interessante considerar a estratégia de oportunizar aos adolescentes a prática de tipos de AF variados, possibilitando aos mesmos a exploração de suas preferências e identificação daquelas atividades que mais lhes são interessantes. Atividades como dança e ginástica não são comumente oferecidas na Educação Física Escolar, e não requerem estruturas adicionais àquelas já disponíveis nas escolas. Nesse sentido, incluir essas atividades nas aulas de Educação Física ou ofertá-las no contra turno (por exemplo, em projetos como o "Mais Educação") é uma alternativa interessante na promoção de AF entre as moças. Ainda, a participação em diferentes tipos de AF pode diferir conforme outros fatores sociodemográficos, como turno de estudo, estrato socioeconômico e acesso aos locais de prática. Portanto, sugere-se que mais investigações sejam realizadas, em diferentes regiões do país, a fim de identificar o perfil de prática de adolescentes brasileiros conforme indicadores socioeconômicos. 


\section{Referências}

AARON, D. J.; STORTI, K. L.; ROBERTSON, R. J.; KRISKA, A. M.; LAPORTE, R. E. Longitudinal study of the number and choice of leisure time physical activities from mid to late adolescence: Implications for School Curricula and Community Recreation Programs. Arch Pediatr Adolesc Med, v. 156, n. 11, p. 1075-80, nov. 2002.

ALVES, J. G. B.; MONTENEGRO, F. M. U.; OLIVEIRA, F. A.; ALVES, R. V. Prática de esportes durante a adolescência e atividade física de lazer na vida adulta. Revista Brasileira de Medicina do Esporte, v. 11, p. 291-294, 2005.

ATHENSTAEDT, U. On the content and structure of the gender role self-concept: Including gender-stereotypical behaviors in addition to traits. Psychology of Women Quarterly, v. 27, n. 4, p. 309-318, dez. 2003.

ATHENSTAEDT, U.; MIKULA, G.; BREDT, C. Gender role self-concept and leisure activities of adolescents. Sex Roles, v. 60, n. 5-6, p. 399-409, mar. 2009.

AZEVEDO JUNIOR, M. R. de; ARAÚJO, C. L. P.; PEREIRA, F. M. Atividades físicas e esportivas na adolescência: mudanças de preferências ao longo das últimas décadas; Physical and sports activities in adolescence: changes in choices over the last decades. Rev. bras. educ. fís. esp, v. 20, n. 1, p. 51-58, 2006.

BARBOSA FILHO, V. C. Fatores comportamentais de risco à saúde cardiovascular em adolescentes de Curitiba, Paraná. 2012. Dissertação (Mestrado em Educação Física)Departamento de Educação Física, Setor de Ciências Biológicas da Universidade Federal do Paraná, Curitiba, 2012.

Atividade física e ambiente escolar: Discutindo práticas e percepções na adolescência. Curitiba: Editora Appris, 2016.

BAUMAN, A. E.; REIS, R. S.; SALLIS, J. F.; WELLS, J. C.; LOOS, R. J.; MARTIN, B. W.; LANCET PHYSICAL ACTIVITY SERIES WORKING GROUP. Correlates of physical activity: why are some people physically active and others not? The lancet, v. 380, n. 9838, p. 258-271, jul. 2012.

BELANGER, M.; GRAY-DONALD, K.; O’LOUGHLIN, J.; PARADIS, G.; HANLEY, J. When Adolescents Drop the Ball: Sustainability of Physical Activity in Youth. Am J Prev Med, v. 37, n. 1, p. 41-9, jul. 2009.

BRINGOLF-ISLER, B.; GRIZE, L.; MÄDER, U.; RUCH, N.; SENNHAUSER, F. H.; BRAUN-FAHRLÄNDER, C. Assessment of intensity, prevalence and duration of everyday activities in Swiss school children: A cross-sectional analysis of accelerometer and diary data. The International Journal of Behavioral Nutrition and Physical Activity, v. 6, n. 50. p. 110, ago. 2009.

BUTTE, N. F.; PUYAU, M. R.; ADOLPH, A. L.; VOHRA, F. A.; ZAKERI, I. Physical Activity in Nonoverweight and Overweight Hispanic Children and Adolescents. Medicine \& Science in Sports \& Exercise, v. 39, n. 8, p. 1257-1266, ago. 2007. 
CAMARGO, L. O. de L. O legado de Joffre Dumazedier: reflexões em memória do centenário de seu nascimento. Revista Brasileira de Estudos do Lazer, Belo Horizonte, v. 3, n. 1, p. 142-66, abr. 2016.

CORDER, K.; WINPENNY, E.; LOVE, R.; BROWN, H. E.; WHITE, M.; SLUIJS, E. van. Change in Physical Activity from Adolescence to Early Adulthood: A Systematic Review and Meta-Analysis of Longitudinal Cohort Studies. British Journal of Sports Medicine, p. 1-9, jul. 2017.

DE BEM, M.; BARROS, M.; OLIVEIRA, E.; LOCH, M.; NAHAS, M. Reprodutibilidade de um questionário para avaliação do estilo de vida e comportamentos de risco de estudantes do ensino médio em Santa Catarina. In: XXIV SIMPÓSIO INTERNACIONAL DE CIÊNCIAS DO ESPORTE, 2000, São Paulo. Anais... São Paulo, 2000. p. 70.

DIAS, G.; CLEMENTE, F.; ROCHA, R.; MARTINS, F.; MARTINS, R.; MENDES, R.; LOPES, A. Estudo dos níveis de atividade física de uma população rural portuguesa. Revista Gymnasium, v. 5, n. 1, p. 1, dez. 2014.

DISHMAN, R. K.; MOTL, R. W.; SAUNDERS, R.; FELTON, G.; WARD, D. S.; DOWDA, M.; PATE, R. R. Enjoyment mediates effects of a school-based physical-activity intervention. Medicine and science in sports and exercise, v. 37, n. 3, p. 478-487, 2005.

DUMAZEDIER, J. Sociologia empírica do lazer. São Paulo: Perspectiva: SESC, 1979.

DUMITH, S. C. Physical activity in Brazil: a systematic review. Cadernos de Saúde Pública, v. 25, p. S415-S426, 2009.

DUMITH, S. C.; DOMINGUES, M. R.; GIGANTE, D. P.; HALLAL, P. C.; MENEZES, A. M. B.; KOHL, H. W. Prevalence and correlates of physical activity among adolescents from Southern Brazil. Revista de Saúde Pública, São Paulo, v. 44, p. 457-67, jun. 2010.

DUMITH, S. C.; GIGANTE, D. P.; DOMINGUES, M. R.; KOHL, H. W. Physical activity change during adolescence: a systematic review and a pooled analysis. International journal of epidemiology, v. 40, n. 3, p. 685-698, 2011.

DUMITH, S. C.; GIGANTE, D. P.; DOMINGUES, M. R.; HALLAL, P. C.; MENEZES, A. M.; KOHL III, H. W. A longitudinal evaluation of physical activity in Brazilian adolescents: tracking, change and predictors. Pediatric exercise science, v. 24, n. 1, p. 58-71, fev. 2012a.

DUMITH, S. C.; MENEZES, A. M. B.; BIELEMANN, R. M.; PETRESCO, S.; SILVA, I. C. M. da; LINHARES, R. da S.; AMORIM, T. C.; DUARTE, D. V.; ARAÚJO, C. L. P.; SANTOS, J. V. dos. Insatisfação corporal em adolescentes: um estudo de base populacional. Ciência \& Saúde Coletiva, v. 17, p. 2499-2505, set. 2012 b.

ESCULCAS, C.; MOTA, J. Actividade física e práticas de lazer em adolescentes. Revista Portuguesa de Ciências do Desporto, v. 5, n.1, p. 69-76, jan. 2005.

FARIA, E. L. Quando "rola a bola": reflexões sobre as práticas futebolísticas e a forma escolar nas aulas de Educação Física. Revista Brasileira de Ciências do Esporte, v. 36, n. 2, 2014. 
FREITAS, R. W. J. F. de; SILVA, A. R. V. da; ARAÚJO, M. F. M. de; MARINHO, N. B. P.; DAMASCENO, M. M. C.; OLIVEIRA, M. R. de. Physical activity practice by adolescents from Fortaleza, CE, Brazil. Revista Brasileira de Enfermagem, v. 63, n. 3, p. 410-415, 2010.

GONÇALVES, V. O.; MARTÍNEZ, J. P. Imagem corporal de adolescentes: um estudo sobre as relações de gênero e influência da mídia. Comunicação \& Informação, v. 17, n. 2, 15 dez. 2014. Disponível em: <http://www.revistas.ufg.br/index.php/ci/article/view/31792>. Acesso em: 18 maio 2018.

GRIESER, M.; VU, M. B.; BEDIMO-RUNG, A. L.; NEUMARK-SZTAINER, D.; MOODY, J.; YOUNG, D. R.; MOE, S. G. Physical Activity Attitudes, Preferences, and Practices in African American, Hispanic, and Caucasian Girls. Health Educ Behav, v. 33, n. 1, p. 40-51, fev. 2006.

JANSSEN, I.; LEBLANC, A. G. Systematic review of the health benefits of physical activity and fitness in school-aged children and youth. International journal of behavioral nutrition and physical activity, v. 7, n. 1, p. 40, 2010.

KJØNNIKSEN, L.; TORSHEIM, T.; WOLD, B. Tracking of leisure-time physical activity during adolescence and young adulthood: a 10-year longitudinal study. International Journal of Behavioral Nutrition and Physical Activity, v. 5, n. 1, p. 69, 2008.

KOORTS, H.; MATTOCKS, C.; NESS, A. R.; DEERE, K.; BLAIR, S. N.; PATE, R. R.; RIDDOCH, C. The Association between the Type, Context, and Levels of Physical Activity amongst Adolescents. J Phys Act Health, v. 8, n. 8, p. 1057-65, nov. 2011.

LANDRY, B. W.; DRISCOLL, S. W. Physical Activity in Children and Adolescents. PM\&R, v. 4, n. 11, p. 826-832, nov. 2012.

LIRA, A. G.; GANEN, A. de P.; LODI, A. S.; ALVARENGA, M. dos S. Uso de redes sociais, influência da mídia e insatisfação com a imagem corporal de adolescentes brasileiras. Jornal Brasileiro de Psiquiatria, v. 66, n. 3, p. 164-171, set. 2017.

LUZ, G. da; KUHNEN, A. O uso dos espaços urbanos pelas crianças: explorando o comportamento do brincar em praças públicas. Psicologia: Reflexão e Crítica, v. 26, n. 3, p. 552560, 2013.

MALTA, D. C.; SARDINHA, L. M. V.; MENDES, I.; BARRETO, S. M.; GIATTI, L.; CASTRO, I. R. R. de; MOURA, L. de; DIAS, A. J. R.; CRESPO, C. Prevalência de fatores de risco e proteção de doenças crônicas não transmissíveis em adolescentes: resultados da Pesquisa Nacional de Saúde do Escolar (PeNSE), Brasil, 2009. Ciênc saúde coletiva, v. 15, n. 2, p. 3009-19, 2010.

MARKLAND, D.; INGLEDEW, D. K. The relationships between body mass and body image and relative autonomy for exercise among adolescent males and females. Psychology of Sport and Exercise, v. 8, n. 5, p. 836-853, 2007.

MATIAS, T. S.; ROLIM, M. K. S. B.; SCHMOELZ, C. P.; ANDRADE, A. Hábitos de atividade física e lazer de adolescentes. Pensar a Prática, Goiânia, v. 15, n. 3, out. 2012. Dispo- 
nível em: <http://www.revistas.ufg.br/index.php/fef/article/view/14744>. Acesso em: 18 maio 2018.

MCMULLAN, S.; CHIN, R.; FROUDE, E.; IMMS, C. Prospective study of the participation patterns of grade 6 and year 8 students in Victoria, Australia in activities outside of school. Australian Occupational Therapy Journal, v. 59, n. 3, p. 197-208, 2012.

MELLOR, D.; HUCKER, A.; WATERHOUSE, M.; BINTI MAMAT, N. H.; XU, X.; COCHRANE, J.; MCCABE, M.; RICCIARDELLI, L. A Cross-Cultural Study Investigating Body Features Associated With Male Adolescents' Body Dissatisfaction in Australia, China, and Malaysia. American Journal of Men's Health, v. 8, n. 6, p. 521-531, nov. 2014.

MELlOR, D.; WATERHOUSE, M.; MAMAT, N. H. bt; XU, X.; COCHRANE, J.; MCCABE, M.; RICCIARDELLI, L. Which body features are associated with female adolescents' body dissatisfaction? A cross-cultural study in Australia, China and Malaysia. Body Image, v. 10, n. 1, p. 54-61, jan. 2013.

MORTON, K. L.; ATKIN, A. J.; CORDER, K.; SUHRCKE, M.; SLUIJS, E. M. F. The School Environment and Adolescent Physical Activity and Sedentary Behaviour: A MixedStudies Systematic Review. Obesity Reviews, v. 17, n. 2, p. 142-158, fev. 2016.

NAHAS, M. V. Atividade Física, Saúde e Qualidade de Vida: Conceitos e Sugestões Para Um Estilo de Vida Ativo. 7. ed. [s.1.] Midiograf, 2017.

OLIVEN, R. A parte e o todo: a diversidade cultural no Brasil-nação. [s.l.] Editora Vozes Limitada, 2011.

ORTEGA, F. B.; KONSTABEL, K.; PASQUALI, E.; RUIZ, J. R.; HURTIG-WENNLÖF, A.; MÄESTU, J.; LÖF, M.; HARRO, J.; BELLOCCO, R.; LABAYEN, I. Objectively measured physical activity and sedentary time during childhood, adolescence and young adulthood: a cohort study. PloS one, v. 8, n. 4, p. e60871, 2013.

PFEIFER, L. I.; MARTINS, Y. D.; SANTOS, J. L. F. A influência socioeconômica e de gênero no lazer de adolescentes. Psicologia: Teoria e Pesquisa, v. 26, n. 3, p. 427-432, set. 2010.

PIRES, E. A. G.; DUARTE, M. de F. da S.; PIRES, M. C.; SOUZA, G. de S. e. Hábitos de atividade física e o estresse em adolescentes de Florianópolis - SC, Br Physical activity habits and stress in teenagers from Florianópolis - SC, Brazil. Rev. bras. ciênc. mov, v. 12, n. 1, p. 51-56, 2004.

RHODES, R. E.; NIGG, C. R. Advancing Physical Activity Theory: A Review and Future Directions. Exercise and Sport Sciences Reviews, v. 39, n. 3, p. 113-119, jul. 2011.

RODGERS, R. F.; MCLEAN, S. A.; PAXTON, S. J. Longitudinal Relationships among Internalization of the Media Ideal, Peer Social Comparison, and Body Dissatisfaction: Implications for the Tripartite Influence Model. Developmental Psychology, v. 51, n. 5, p. 706-713, maio 2015. 
ROLEY, S. S.; BARROWS, C. J.; SUSAN BROWNRIGG OTR, L.; SAVA, D. I.; VIBEKE TALLEY OTR, L.; KRISTI VOELKERDING, B.; COTA, L.; MOT, E. S.; PAMELA TOTO, M.; DEBORAH LIEBERMAN, M. Occupational therapy practice framework: Domain \& process 2nd edition. The American journal of occupational therapy, v. 62, n. 6, p. 625, 2008.

SALLES-COSTA, R.; HEILBORN, M. L.; WERNECK, G. L.; FAERSTEIN, E.; LOPES, C. S. Gênero e prática de atividade física de lazer. Cadernos de Saúde Pública, v. 19, p. S325S333, 2003.

SANTOS, M. P.; GOMES, H.; RIBEIRO, J. C.; MOTA, J. Variação sazonal na actividade física e nas práticas de lazer de adolescentes portugueses. Revista Portuguesa de Ciências do Desporto, v. 5, p. 192-201, 2005.

SEABRA, A. F.; MENDONÇA, D. M.; THOMIS, M. A.; ANJOS, L. A.; MAIA, J. A. Determinantes biológicos e sócio-culturais associados à prática de atividade física de adolescentes. Cad. saúde pública, v. 24, n. 4, p. 721-36, 2008.

SIGMUNDOVA, D.; EL ANSARI, W.; SIGMUND, E.; FROMEL, K. Secular trends: A tenyear comparison of the amount and type of physical activity and inactivity of random samples of adolescents in the Czech Republic. BMC Public Health, v. 11, p. 731, 2011.

SILVA, I.; MIELKE, G.; NUNES, B.; BÖHM, A.; BLANKE, A.; NACHTIGALL, M.; CRUZ, M.; MARTINS, R.; MÜLLER, W.; HALLAL, P. Espaços públicos de lazer: distribuição, qualidade e adequação à prática de atividade física. Revista Brasileira de Atividade Física \& Saúde, v. 20, n. 1, p. 82, 2015.

SILVA, K. S.; LOPES, A. da S.; HOEFELMANN, L. P.; CABRAL, L. G. de A.; DE BEM, M. F. L.; BARROS, M. V. G. de; NAHAS, M. V. Health risk behaviors Project (COMPAC) in youth of the Santa Catarina State, Brazil: ethics and methodological aspects. Revista Brasileira de Cineantropometria \& Desempenho Humano, v. 15, n. 1, jan. 2013.

SOUSA, T. F. de. Atividades físicas praticadas no lazer por universitários de uma instituição pública do nordeste do Brasil. Rev. bras. ativ. fís. saúde, v. 17, n. 2, 2012.

STERDT, E.; LIERSCH, S.; WALTER, U. Correlates of physical activity of children and adolescents: A systematic review of reviews. Health Education Journal, v. 73, n. 1, p. $72-$ 89, jan. 2014.

WOLL, A.; KURTH, B. M.; OPPER, E.; WORTH, A.; BOS, K. The "Motorik-Modul" (MoMo): Physical fitness and physical activity in German children and adolescents. Eur $\mathbf{J}$ Pediatr, v. 170, n. 9, p. 1129-42, set. 2011.

ZICK, C. D. The shifting balance of adolescent time use. Youth \& Society, v. 41, n. 4, p. 569-596, 1 jun. 2010. 
Endereço para correspondência:

marcusvvl@hotmail.com

Marcus Vinicius Veber Lopes

Universidade Federal de Santa Catarina

Departamento de Educação Física -Campus Universitário,

Trindade 88040-900, Florianópolis, SC 